Published in final edited form as: IEEE Trans Ultrason Ferroelectr Freq Control. 2005 September ; 52(9): 1608-1612.

\title{
Characterization of very high frequency transducers with wire target and hydrophone
}

\author{
Bin Huang and $\mathrm{K}$ Kirk Shung \\ Dept. of Biomedical Engineering, University of Southern California, Los Angeles, CA, 90089
}

\section{Abstract}

In this paper, the wire-target technique will be shown to be a useful alternative for beam profile measurements in very high frequency range $(30-60 \mathrm{MHz})$. A $9 \mathrm{~cm}$ long tungsten wire with a diameter of $8 \mu \mathrm{m}$ was used as the pulse-echo target to measure the lateral beam profiles at the focal points of two transducers, a spherically focused $40 \mathrm{MHz}$ Panametrics transducer with an aperture size of 6.35 $\mathrm{mm}$ and a geometrical focal length of $12.7 \mathrm{~mm}$ and a lense-focused in-house lithium niobate $\left(\mathrm{LiNbO}_{3}\right) 60 \mathrm{MHz}$ transducer with an aperture size of $2 \mathrm{~mm}$ and a geometrical focal length around $6.5 \mathrm{~mm}$. For comparison, measurements on the same transducers were performed by three smallaperture hydrophones. The first one is a polyvinylidene fluoride trifluoroethylene (PVDF-TrFE) membrane hydrophone developed by Hewlett-Packard, which has a geometric diameter of $37 \mu \mathrm{m}$, a measured effective diameter of less than $100 \mu \mathrm{m}$ and a $-3 \mathrm{~dB}$ bandwidth of more than $150 \mathrm{MHz}$. The second one is a needle-type PVDF hydrophone from Precision Acoustics, which has a $9 \mu \mathrm{m}-$ thick PVDF element, a $40 \mu \mathrm{m}$ geometrical aperture and a measured effective diameter of less than $100 \mu \mathrm{m}$. The third one is a needle-type PVDF hydrophone from Onda, which has a 150- $\mu \mathrm{m}$ geometrical aperture and an effective diameter of about $180 \mu \mathrm{m}$. Experimental results show that the $-6 \mathrm{~dB}$ two-way beam widths measured by this $8 \mu \mathrm{m}$ wire-target are in agreement with $-3 \mathrm{~dB}$ transmitted beam widths measured by small-aperture hydrophones. Compared to small-aperture hydrophones, the wire-target technique is simpler and more cost-effective. Its major advantage however is in the frequency range above $100 \mathrm{MHz}$ in which commercial hydrophones are not yet available.

\section{Introduction}

Very high frequency ultrasonic imaging with medical applications in dermatology, ophthalmology and intravascular imaging, and small animal imaging has been speculated by many to be the next frontier in ultrasonic imaging. Though the hydrophone method $[1,2]$ is considered the gold standard for the characterization of transducers, conventional hydrophones often suffer severely from the effect of spatial averaging and often cannot meet the requirements of charactering high frequency transducers. Small aperture hydrophones that reduce spatial averaging are expensive and have limitations on the frequency range.

Instead of the hydrophone method, a simple wire-target technique proposed by Raum and O'Brien [3] was used in this research to characterize very high frequency transducers. Using a wire-target as the pulse-echo target, translational scans at the focus of the transducer yield the lateral profile of projections of the beam. For a symmetric beam with relative smallside lobes, the line integral projections closely track the point-to-point measurement. Raum and O'Brien [3] gave results in the frequency range of 3-17 MHz. In this research, we extended the wire-target technique to the frequency range of 35-60 MHz. The results are compared to measurements from small aperture hydrophones.

E-mail:kkshung@usc.edu. 


\section{Experiment}

The experimental system was comprised of a wire-target, three small aperture hydrophones and a computer-controlled exposimetry system designed in house [6], which includes a water tank filled with deionized water, a digitizing (500 MS/s) oscilloscope (Model 534, Lecroy Corp., Chestnut Ridge, NY), a three-axis positioning system (Burleigh Inc., Fishers, NY) with a resolution of about $0.5 \mu \mathrm{m}$, as shown in Figure 1 .

The wire-target was made from a $9 \mathrm{~cm}$ tungsten wire with a diameter of $8 \mu \mathrm{m}$ (California Fine Wire, Grover Beach, CA).

Also three small-aperture hydrophones were used for comparison. The first one is a PVDFTrFE membrane hydrophone [4] ]. This hydrophone was fabricated on a 4- $\mu$ m-thick film of PVDF-TrFE, with a $-3 \mathrm{~dB}$ bandwidth in excess of $150 \mathrm{MHz}$. It has a geometric spot diameter of $37 \mu \mathrm{m}$, a measured effective spot diameter of less than $100 \mu \mathrm{m}$ and an on-membrane $7 \mathrm{~dB}$ gain buffer amplifier. The second hydrophone is a needle-type PVDF hydrophone (Precision Acoustics, Dorchester, UK). It has a $40 \mu \mathrm{m}$ geometrical aperture, a $9 \mu \mathrm{m}$-thick PVDF element and a $12 \mathrm{~dB}$ submersible pre-amplifier. An external $25 \mathrm{~dB}$ amplifier with a bandwidth of 125 $\mathrm{MHz}$ was used along with those two hydrophones. The third one is a needle-type PVDF hydrophone from Onda (Onda, Sunnyvale, CA), which has a 150- $\mu \mathrm{m}$ geometrical aperture and an effective diameter of about $180 \mu \mathrm{m}$. Table 1 lists major characteristics of those smallaperture hydrophones.

Two transducers were used in the experiments. A spherically focused $40 \mathrm{MHz}$ Panametrics transducer with an aperture size of $6.35 \mathrm{~mm}$ and a geometrical focal length of $12.7 \mathrm{~mm}$ and a lens-focused $\mathrm{LiNbO}_{3} 60 \mathrm{MHz}$ transducer with an aperture size of $2 \mathrm{~mm}$ and a geometrical focal length around $6.5 \mathrm{~mm}$ fabricated in house. The transducers were excited by the Panametrics pulser/receiver (Model 5900PR and 5910RPP, Panametrics, Waltham, MA). For the pulseecho signal from wire-target, the echo was amplified and band pass filtered by the pulser/ receiver system before displayed on oscilloscope.

During the experiment, wire-target/hydrophones were placed near the focus of the transducers and the wire-target or a hydrophone was scanned by the positioning system perpendicular to acoustic axis of the transducer to obtain a lateral beam profile. The step size was $5 \mu \mathrm{m}$ for the wire-target in all measurements. For hydrophone measurements, the step size was $25 \mu \mathrm{m}$ for $40 \mathrm{MHz}$ Panametrics transducer and $15 \mu \mathrm{m}$ for $60 \mathrm{MHz} \mathrm{LiNbO}_{3}$ transducer.

\section{Results and Discussions}

The hydrophone-determined lateral beam profiles were compared to the wire-determined beam profile (Figures 2 and 3). In these figures, the normalized transmit lateral beam profiles measured by hydrophones were multiplied by 2 for comparison to the pulse-echo beam profile measured with the wire-target. The two way $-3 \mathrm{~dB}$ beam widths from hydrophones and $-6 \mathrm{~dB}$ transmit-receive beam width from the wire-target are given in Table 2 . The theoretical value of the beam width is also shown at the bottom row of the table. Table 3 shows the center frequency and $-20 \mathrm{~dB}$ pulse duration obtained around the focus of the transducers. All acoustic field definitions can be found on [2].

With its broad bandwidth and smallest aperture size, HP membrane hydrophone suffers less from the spatial averaging. For both transducers, the beam width from HP hydrophone is the smallest and the pulse duration ( $36.4 \mathrm{~ns}$ ) for $60 \mathrm{MHz}$ transducer the shortest. However, the signal to noise ratio of HP membrane hydrophone is relatively poor. In Figure 2 and Figure 3, it can be seen that due to noise, there are irregularities in lateral beam profiles measured with the HP hydrophone when the lateral position is away from the center. 
The Onda hydrophone has higher signal to noise ratio, but it has the largest spatial averaging effects due to its largest effective diameter (about $180 \mu \mathrm{m}$ ). When measuring the $60 \mathrm{MHz}$ transducer, the center frequency obtained from Onda hydrophone $(40.7 \mathrm{MHz})$ differed substantially from those estimated by other devices.

The wire-target method is the simplest. It has the highest signal to noise ratio though its results may not be as "ideal" as those obtained by HP membrane hydrophone. In fact the utilization of a smaller diameter wire-target may further improve the results because of a smaller spatial averaging effect presented by the area of the wire. It can certainly be extended to the frequency range above $100 \mathrm{MHz}$. The wire-target method in a sense is analogous to the optical Schlieren method. Tomographic reconstruction algorithms can be used to remove the line integral effect. In order to do so while achieving satisfactory resolution, 180 projections may be needed. One projection will need 5 10 minutes. Thus, for 180 projections $15 \sim 30$ hours may be needed. In such a case, it is even more time-consuming than the point-to-point hydrophone measurements.

Small aperture hydrophones have directivity. The higher the frequency, the stronger of the directivity. The directivity of wire target is complex, and also depends on the wavelength. More research is needed to further address the effect of this issue on the accuracy of the results.

\section{Conclusion}

The results reported in this paper demonstrate that the wire-target technique is a useful alternative to small-aperture hydrophones in characterizing ultrasonic transducer radiation patterns. The wire-target technique is simpler and more cost-effective. Its major advantage however is in the frequency range above $100 \mathrm{MHz}$ in which commercial hydrophones are not yet available.

\section{Acknowledgements}

This work was supported by NIH grant \#P41-EB2182.

\section{References}

1. IEEE. IEEE guide for medical ultrasound field parameter measurements. IEEE 19901989:790.

2. AIUM 1992 Acoustic Output Measurement and Labeling Standard for Diagnostic Ultrasound Equipmenmt (Laurel,MD)

3. Kay, Raum; O'Brien, WD. Pulse-Echo Field Distribution Measurement Technique for High-Frequency Ultrasound Sources. IEEE Trans Ultrason Ferroelec Freq Contr 1997;44(4):810-815.

4. Lum P, Greenstein M, Grossman C, Szabo TL. High-freqency membrane hydrophone. IEEE Trans Ultrason Ferroelec Freq Contr July;1996 43(4):536-44.

5. Lum, P.,Greenstein,M.etc. A 150-MHz-Bandwidth Membrane Hydrophone for Acoustic Field Characterization, The Hewlett-Packard Journal, 6-16 August 1988

6. Snook KA, Huang B, Smith NB, Shung KK. An exposimetry system for characterization of acoustic fields above $20 \mathrm{MHz}$. 2000 IEEE Ultrasonics Symposium 2:1109-12. 




Figure 1.

Block diagram of the experimental arrangement. 


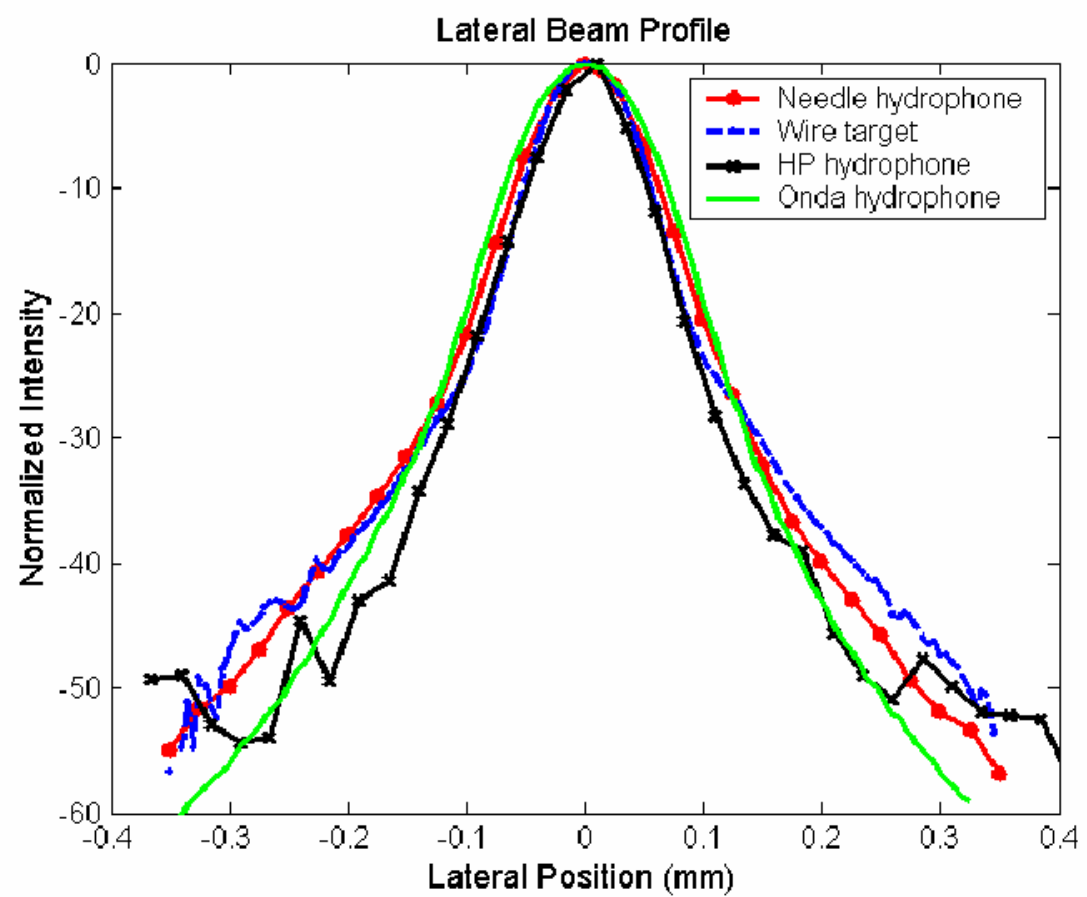

Figure 2.

Lateral beam profiles of the $40 \mathrm{MHz}$ Panametrics transducer 


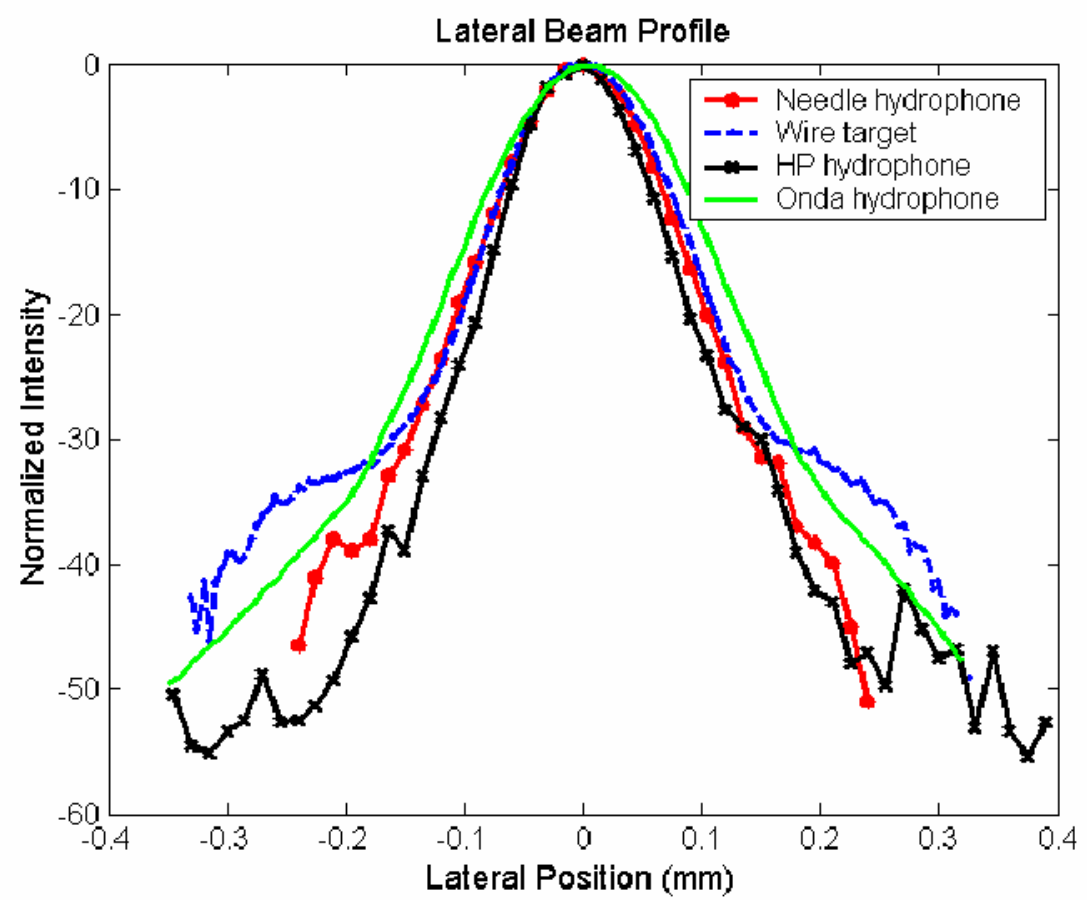

Figure 3.

Lateral beam profiles of the $60 \mathrm{MHz} \mathrm{LiNbO} 3$ transducer 
Table 1

Major characteristics of the three small aperture hydrophones

\begin{tabular}{lccc}
\hline & PA needle hydrophone & HP membrane hydrophone & Onda Needle hydrophone \\
\hline Manufacturer & Precision Acoustics Limited & Hewlett-Packard & Onda \\
Piezoelectric material & PVDF & PVDF-TrFE & PVDF \\
Geometrical Diameter & $40 \mu \mathrm{m}$ & $37 \mu \mathrm{m}$ & $150 \mu \mathrm{m}$ \\
Effective Diameter & $<100 \mu \mathrm{m}$ & $<100 \mu \mathrm{m}$ & $4 \mu \mathrm{m}$ \\
Film thickness & $9 \mu \mathrm{m}$ & $7 \mathrm{~dB}$ & $\sim$ \\
On-membrane Amplifier & $12 \mathrm{~dB}$ & & $\sim$ \\
& & & \\
\hline
\end{tabular}


Table 2

$-6 \mathrm{~dB}$ transmit-receive beam widths measured by $8 \mu \mathrm{m}$ wire target and $-3 \mathrm{~dB}$ transmit beam widths measured by three small-aperture hydrophones

\begin{tabular}{|c|c|c|c|}
\hline$Z$ & & $\begin{array}{l}\text { Panametrics transducer Beam width } \\
\qquad(\mu \mathrm{m})\end{array}$ & In-House transducer Beam width $(\mu \mathrm{m})$ \\
\hline & $8 \mu \mathrm{m}$ wire target & 82.3 & 107.1 \\
\hline & HP Hydrophone & 77.4 & 92.4 \\
\hline$D$ & PA Hydrophone & 89.6 & 103 \\
\hline$£$ & Onda Hydrophone & 104.7 & 126.4 \\
\hline $\overrightarrow{\overrightarrow{2}}$ & f-number $\times$ wavelength & 74.1 & 80 \\
\hline
\end{tabular}


Table 3

Center frequency and pulse duration measured by wire target and small aperture hydrophones

\begin{tabular}{|c|c|c|c|c|}
\hline & \multicolumn{2}{|c|}{ Panametrics transducer } & \multicolumn{2}{|c|}{ In-House transducer } \\
\hline & Center frequency $(\mathrm{MHz})$ & Pulse duration (ns) & Center frequency $(\mathrm{MHz})$ & Pulse duration (ns) \\
\hline $8 \mu \mathrm{m}$ wire target & 42.5 & 64.9 & 56.2 & 55.7 \\
\hline HP Hydrophone & 40.6 & 49 & 60.3 & 36.4 \\
\hline PA Hydrophone & 37.5 & 51.3 & 58.4 & 53.2 \\
\hline Onda Hydrophone & 34.7 & 49.2 & 40.7 & 53 \\
\hline
\end{tabular}

